\title{
Processes of isothermal formation of hollow t-shaped tubes made of high-strength alloys
}

\author{
Andrey A. Pasynkov* , Sergey N. Larin, and Lydmila P. Semenova \\ Tula state university, 300012 Lenina ave., 92, Russia
}

\begin{abstract}
Slow hot deformation makes it possible to achieve a significant growth of deformation without the destruction of the material and avoiding a significant decrease in pressure when forming difficult-to-deform nonferrous alloys. This is especially important when it is necessary to obtain products that operate in severe conditions, for example, in propulsion systems. The article discusses the manufacture of hollow T-shaped connectors by means of forming, namely, by means of extrusion in orthogonal planes and piercing. It was assumed that these operations took place under isothermal conditions. Expressions for calculating the force values of the processes are obtained. Using these expressions, the influence of the degrees of deformation on the forces of extrusion and piercing was investigated.
\end{abstract}

\section{Introduction}

In piping systems of power plants, T-shaped tubes are used to connect heat pipes [1-4]. Their operating conditions determine the requirements for materials and their properties for the manufacture of these T-tubes. They are mainly made from resistant non-ferrous alloys that resist treatment [5-9]. The deformation of such alloys in a special viscoplastic mode looks very promising. Hydraulic press in split dies, providing forming in orthogonal planes, can be used as the equipment that allows implementing this mode $[1,2,8,10]$.

An important value here is the rate of deformation [11-14], which ultimately affects the intensity of the deformation. In this article, two particular operations for obtaining hollow T-shaped tubes are considered: orthogonal extrusion and piercing.

To determine the pressures, we will use the energy calculation methods [1-3]. The scheme of operations will be assumed to be axisymmetric.

\section{Main part}

Extrusion in orthogonal planes. Fig. 1, a shoes the extrusion scheme in orthogonal planes and the velocity field. The field includes deformation zones " 2 " and rigid elements " 0 ", "1", "3", which are separated from each other by lines " 01 ", "12", "23" and are limited by the body of the working tool. The state of the metal during extrusion is considered to be viscoplastic. The equation of its state is represented as follows [1, 2]:

* Corresponding author: sulee@mail.ru 


$$
\sigma_{e}=A \varepsilon_{e}^{m} \xi_{e}^{n},
$$

where $\sigma_{e}, \varepsilon_{e} \xi_{e}$ are the intensity values of stresses, strains, strain rates; $A, m, n$ are the constants.

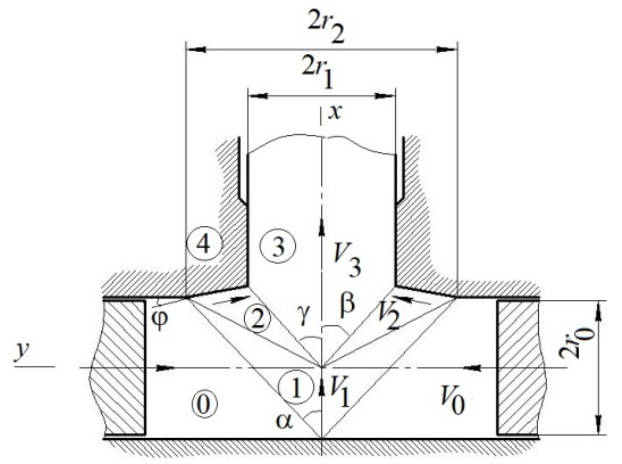

a

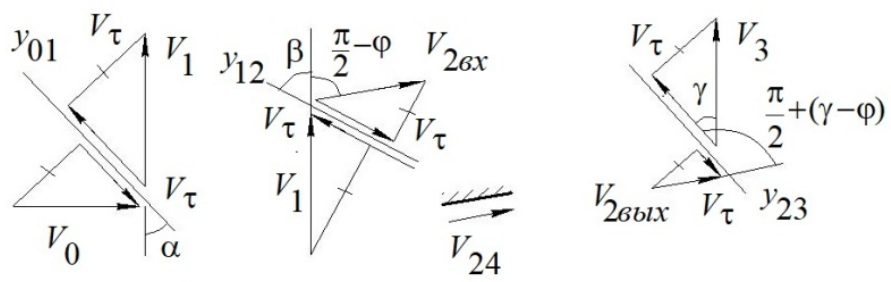

b

Fig. 1. Scheme of the extrusion, field (a) and plan (b) of movement velocities

Using the energy calculation methods [4], we obtained the expressions for calculation of powers in various elements of the selected velocity field [2].

The deformation block's power is [2]

$$
N_{1}=\int_{W} \sigma_{e} \xi_{e} d W=2 \pi A y_{g r . c .}\left(\frac{\Delta h}{V_{0}}\right)^{m} \int_{0}^{c} \int_{y 12}^{y 23} \xi_{e}^{1+m+n} d y d x,
$$

where $W, y_{\text {gr.c. }}$ are respectively the volume of the block and the transverse center of gravity of its cross-sectional area.

The power on the considered lines of velocity gaps is [2]:

$$
N_{p}=\sum \tau V_{\tau} S_{p}=\pi A\left(\frac{2}{\sqrt{3}}\right)^{1+m+n}\left(\frac{\Delta h}{V_{0}}\right)^{m} \sum y_{g r . c .} l_{p}^{1-m-n} V_{\tau}^{1+m+n} .
$$

Here $S_{p}$ is the area of the velocity gap's surfaces; $V_{\tau}$ - shear velocities (9); $l_{p}$ - line lengths (10); $y_{\text {gr.c. }}=\frac{r_{2}}{2} ; y_{\text {gr.c. }}=\frac{r_{2}}{2} ; y_{\text {gr.c. }}=\frac{r_{1}}{2}$ are the transverse centers of gravity of the lines, respectively.

At the contact zone between the tool and the material of the workpiece, the power is:

$$
N_{k}=N_{24}=\pi A y_{u . m .} l_{24}^{-m-n}\left(\frac{2}{\sqrt{3}}\right)^{1+m+n}\left(\frac{\Delta h}{V_{0}}\right)^{m} \int_{r_{0}}^{c}\left[V_{2}(x)\right]_{y=y_{24}}^{1+m+n} d x
$$


where

$$
y_{\text {gr.c. }}=\frac{r_{1}+r_{2}}{2} ; l_{24}=\frac{r_{2}-r_{1}}{\sin \gamma}
$$

The friction power on the contact lines between the tool and the workpiece is [2]:

$$
N_{m p .}=N_{24}=\mu q\left(V_{0} S_{1}+V_{3} S_{2}\right) .
$$

Here $V_{3}=2 V_{0}\left(\frac{r_{0}}{r_{1}}\right)^{2}$ is the value of the velocity of the edge elements of the material at the output end of the tool; $S_{1}, S_{2}$ are the friction zones at the input and output areas of the tool; $\mu$ is the Coulomb friction coefficient.

Taking into account formulas (2-5), the value of the extrusion pressure in orthogonal planes is [2]:

$$
q \leq \frac{N_{1}+N_{p}+N_{k}}{2 \pi r_{0} V_{0}-\mu\left(V_{0} S_{1}+V_{2} S_{2}\right)} .
$$

To find the pressure substituting formulas (2-5) into expression (6), a set of calculations was performed. The values of the constants $A, m, n$ are represented in the table 1 . They were selected from reference books and refer to materials $6 \mathrm{Al}-4 \mathrm{~V}$ and AA5086. Here are the calculations of the following values of basic geometric values: $r_{1}=6 \ldots 20 \mathrm{~mm} ; r_{0}=20$ $\mathrm{mm}, V_{0}=1 \ldots 40 \mathrm{~mm} / \mathrm{min}$.

Table 1. Material constants

\begin{tabular}{|c|c|c|c|c|}
\hline Material & $T,{ }^{\circ} \mathrm{C}$ & $A, M p a \cdot c^{-1}$ & $m$ & $n$ \\
\hline AA5086 & 450 & 55 & 0,1 & 0,025 \\
\hline 6Al-4V & 930 & 67 & 0,03 & 0,06 \\
\hline
\end{tabular}

Fig. 2 shows the dependence for the calculation of the change in pressure during extrusion in orthogonal planes depending on the relative value of the diameter of the extruded part for the materials under consideration.

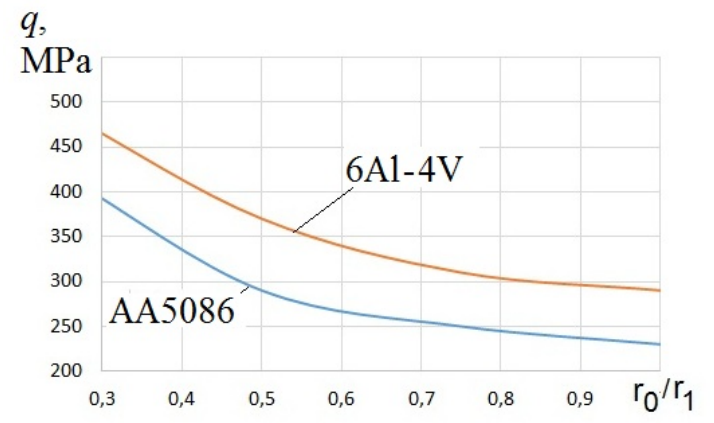

Fig. 2. Graphs $q\left(r_{0} / r_{1}\right)$ for the extrusion in orthogonal planes $\left(V_{0}=1 \mathrm{~mm} / \mathrm{min}\right)$ 
An increase in the relative value of the extrusion diameter for alloy AA5086 results in a $65 \%$ reduction of pressures. For alloy $6 \mathrm{Al}-4 \mathrm{~V}$ it leads to a $50 \%$ decrease of the pressures on the punches.

Fig. 3 shows the dependence, which makes it possible to reveal the change in the pressure of the bar extrusion in the orthogonal planes, depending on the relative pressing punch stroke.

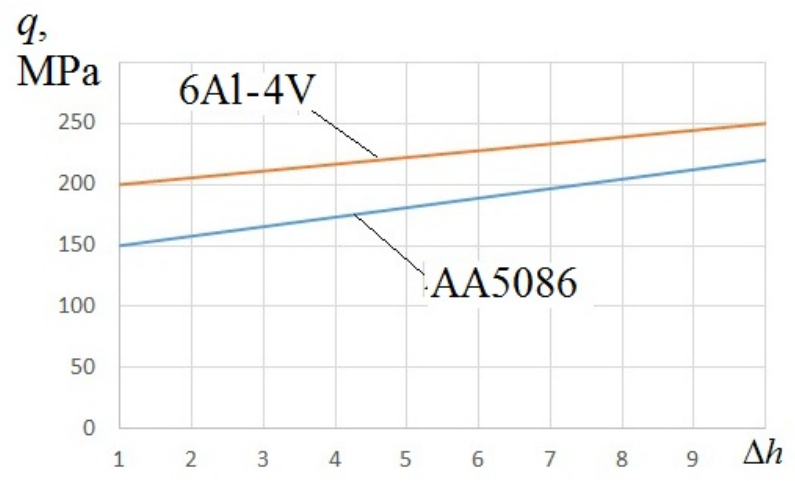

Fig. 3. Graphs $q(\Delta h)$ for extrusion in orthogonal planes $\left(V_{0}=1 \mathrm{~mm} / \mathrm{min}\right)$

An increase in the relative strokes of the deforming punches for titanium $6 \mathrm{Al}-4 \mathrm{~V}$ leads to an increase in pressures on the deforming tool by $20 \%$, and for aluminum AA5086 - by $45 \%$.

Fig. 4 shows the dependence that reveals the change in the bar extrusion pressure in orthogonal planes with an increase of the Coulomb friction coefficient.

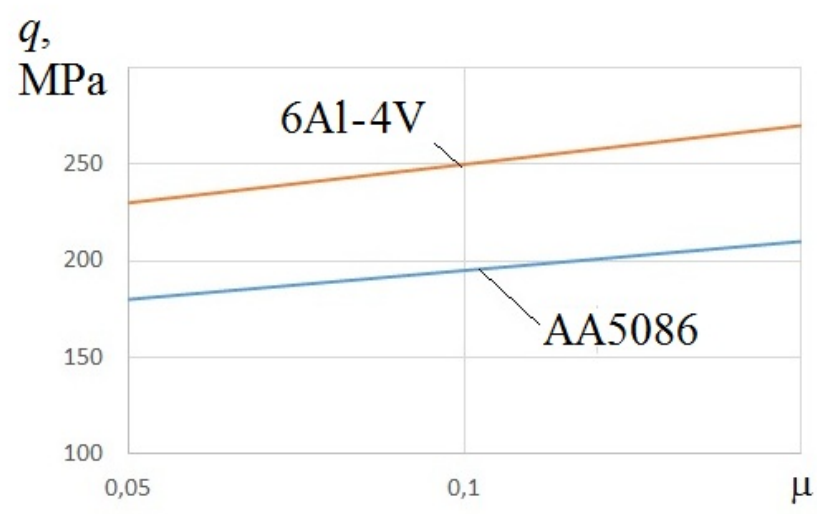

Fig. 4. Graphs for the extrusion of a bar in orthogonal planes

The growth of Coulomb friction coefficient leads to an increase in pressures on the deforming tool by $15 \%$ for titanium $6 \mathrm{Al}-4 \mathrm{~V}$, and for aluminum AA5086 - by $17 \%$.

Isothermal piercing. Piercing operation is used for the formation of holes in solid metal bodies. It is the second stage in the cycle of operations for the manufacture of connectors after the extrusion of the bar in orthogonal planes. Fig. 5. shows the scheme of the analyzed process. 

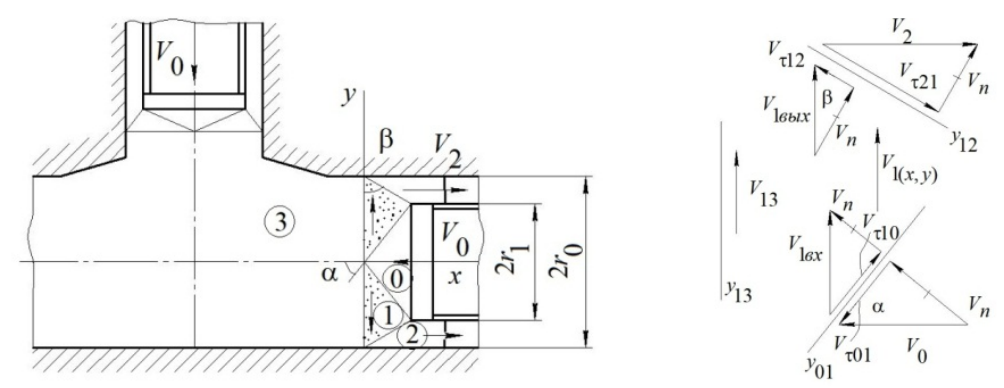

Fig. 5. Scheme of the analyzed process and velocity hodograph

The field of the investigated operation is assumed to be axisymmetric. It includes the block of deformations " 1 " and rigid blocks " 0 ", " 2 ", " 3 ", separated from each other by lines " $01 "$, " $12 "$, " “13".

Using the energy calculation methods [3], we obtained the expressions for calculating the powers in various elements of the selected velocity field [3].

The power of the internal forces in the deformation block is:

$$
N_{1}=2 \pi A y_{\text {gr.c. }}\left(\frac{\Delta h}{V_{0}}\right)^{m} \int_{0}^{h} \int_{y_{01}}^{y 12} \xi_{e}^{1+m+n} d y d x
$$

where $y_{\text {gr.c.. }}$ - is the transverse center of gravity of the sectional area of the block; $\Delta h$ is the working stroke.

The powers of the internal forces on lines " 01 " and " 12 " are [3]:

$$
\begin{aligned}
& N_{01}=\frac{1}{\sqrt{3}} \sigma_{e 01} V_{\tau 01} S_{01}=\pi A r_{1}\left(\frac{1+\operatorname{tg}^{2} \alpha}{\sqrt{3}} \operatorname{ctg} \alpha\right)^{1+m+n}\left(\frac{1}{\Delta h}\right)^{n} V_{0}^{1+n} ; \\
& N_{12}=\frac{1}{\sqrt{3}} \sigma_{e 12} V_{\tau 12} S_{12}=\pi A r_{1}^{2}\left[\frac{1}{\sqrt{3}}\left(1+\frac{\operatorname{ctg} \beta}{\sin \beta}\right) \operatorname{tg} \beta\right]^{1+m+n}\left(\frac{1}{\Delta h}\right)^{n} V_{0}^{1+n} .
\end{aligned}
$$

The powers of the internal forces on line "13" is [3]:

$$
N_{13}=\frac{1}{2} \pi A\left(\frac{2}{\sqrt{3}}\right)^{1+m+n} r_{0}^{2-m-n} \int_{0}^{r 0}\left[V_{1 \mid x=0}(y)\right]^{1+m+n} d y .
$$

The friction power of workpiece material is [3]:

$$
N_{m p .}=\mu q\left[\left(V_{0}+\frac{r_{1}^{2} V_{0}}{r_{0}^{2}-r_{1}^{2}}\right) S_{n}+\frac{r_{1}^{2} V_{0}}{r_{0}^{2}-r_{1}^{2}} S_{\mathcal{M}}\right] .
$$

Here $q$ is the piercing pressure; $S_{n}, S_{M}$ are the areas of friction surfaces on the punch and the die.

The maximum piercing pressure is [3]:

$$
q \leq \frac{N_{1}+N_{01}+N_{12}+N_{13}}{2 \pi r_{1}^{2} V_{0}-\mu\left[\left(V_{0}+V_{2}\right) S_{n}+V_{2} S_{M}\right]} .
$$

To find the pressure substituting formulas (7-11) into expression (12), a set of calculations was performed. The values of the constants $A, m, n$ are listed in the table.

The following geometric values were used for the calculations: $r_{1}=10 \ldots .22 \mathrm{~mm}$; $r_{0}=25 \mathrm{~mm}, \Delta h=10 \mathrm{~mm}$. 
After performing the calculations, the piercing pressure was estimated depending on the degrees of deformation and the velocity of piercing punches for alloys AA5086 and 6Al$4 \mathrm{~V}$. Their temperature modes were $450^{\circ} \mathrm{C}$ and $930^{\circ} \mathrm{C}$ respectively.

Fig. 6 shows the obtained dependences of the pressure change during the piercing, depending on the speed of movement of the piercing punches for the investigated nonferrous alloys.

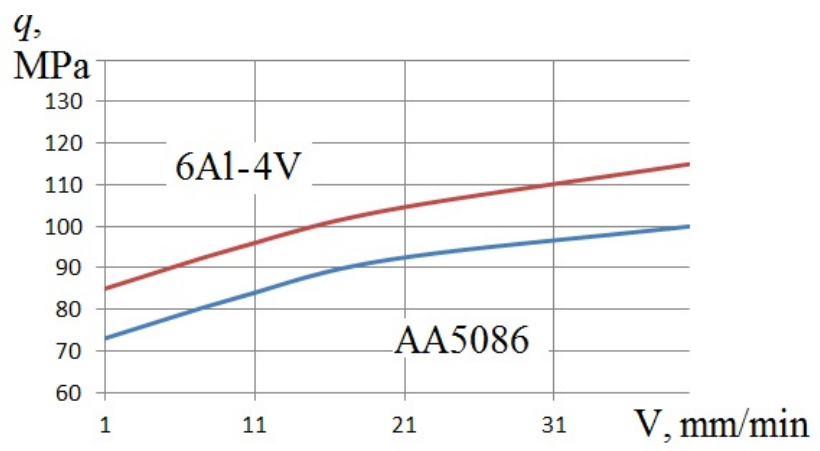

Fig. 6. Dependence of the piercing pressure change on the velocity of punches

According to Fig. 6, with an increase in the velocities of piercing punches' movement, a $27 \%$ increase in pressure on them is observed for the AA5086 alloy and 35\% increase - for the $6 \mathrm{Al}-4 \mathrm{~V}$ alloy. Fig. 7 shows the obtained dependences of the change in pressure during piercing, depending on the relative value of the diameter of the pierced hole.

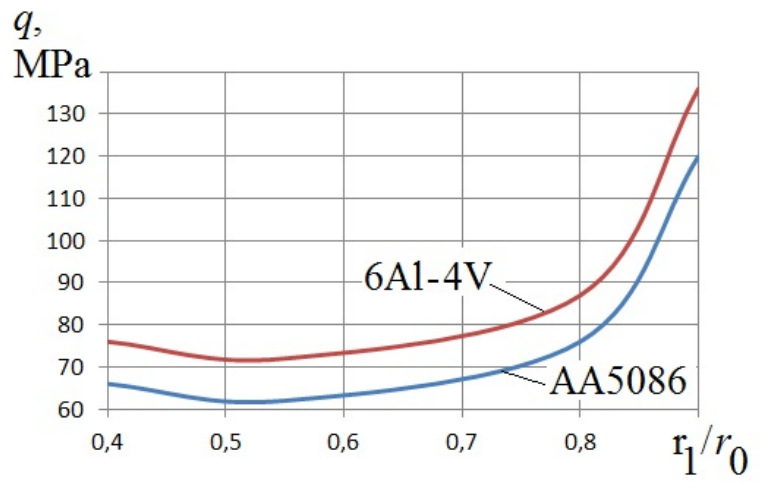

Fig. 7. Dependence of the piercing pressure change on the relative value of the hole diameter

The growth of the relative value of the pierced hole diameter, according to Fig. 6, leads to an increase in pressures on the deforming punches by $1.9 \ldots 2.1$ times for the alloys under study. It should be said that the lines of pressure change first demonstrate a decrease in their values by $5 \%$, and then a 2 -fold increase.

\section{Conclusion}

This mathematical modeling made it possible to obtain expressions describing the power parameters of the T-shaped tubes' production from high-strength alloys. On the basis of the modeling results, the studies of the influence of the deformation on the pressure of the processes were carried out. The studies have confirmed their significance for the growth of 
deformation forces. The results can be used to create practical methods for the design of technologies for the production of hollow T-shaped tubes.

The work was carried out within the framework of the grant of the rector of Tula State University and grant NSH-2601.2020.8.

\section{References}

1. A.A. Pasynkov, S.N. Larin, G.A. Nuzhdin, Evaluation of possibilities of manufacturing by indirect extrusion of cylindrical box-type products from difficult-todeform nonferrous alloys, Non-ferrous Metals. №48. pp. 49-51. (2020)

2. A.A. Pasynkov, Orthogonal isothermal extrusion of parts with lateral processes, Forging and stamping production, Metal forming (to be published).

3. A.A. Pasynkov, Orthogonal isothermal piercing of holes in tees, Forging and stamping production, Metal forming (to be published).

4. Chul Park, Joong-Yeon Lim, Beong-Bok Hwang, A process-sequence design of an axle-housing by cold extrusion using thick-walled pipe, Journal of Materials Processing Technology, v.75, pp. 33-44. (1998)

5. Raj Balendra, Yi Qin, Injection forging: engineering and research, Journal of Materials Processing Technology, v145, pp. 186-206. (2004)

6. Shengfa Zhua, Xincun Zhuanga, Dongkai Xucd, Yin Zhua, Zhen Zhao Flange forming at an arbitrary tube location through upsetting with a controllable deformation zone, Journal of Materials Processing Technology, v273, 116230. (2019).

7. L.M. Alves, R.M. Afonso, C.M.A. Silva, P.A.F. Martins, Joining tubes to sheets by boss forming and upsetting, Journal of Materials Processing Technology, pp. 773781. (2018)

8. Anees Al-Tamimia, Rooholamin Darvizeha, Keith Davey Experimental, Investigation into finite similitude for metal forming processes, Journal of Materials Processing Technology, v.262, pp. 622-637. (2018).

9. $\quad \mathrm{Su}$ Y.H. Frank, Y.C. Chen, Y.A. Tsao Chi, Workability of spray-formed 7075 Al alloy reinforced with SiCp at elevated temperatures, Materials Science and Engineering, v364, pp. 296-304. (2004).

10. A.A. Pasynkov, O.I. Boriskin, S.N. Larin, Theoretical studies of the operation of isothermal distribution of pipes from hard-deformed non-ferrous spaves under shortterm creep conditions, Non-ferrous metals, v2, pp. 74-78. (2018).

11. A.A. Pasynkov, OS.N. Larin, Analysis of forming properties during the isothermal upsetting of cylindrical workpieces in the viscous-plasticity mode, IOP Conference Series: Materials Science and Engineering, (v441). (2018).

12. John Monaghan, Application of the $2 D$ finite element method to simulation of coldforging processes, Journal of Materials Processing Technology, (v77), pp. 305-313. (1998).

13. Y.S. Lee, S.K. Hwang, Y.S. Chang, B.B. Hwang, The forming characteristics of radial-forward extrusion, Journal of Materials Processing Technology, (v113), pp. 136-140. (2001).

14. Su-Hai Hsiang, Chao-Shun Liao Study on hot extrusion of tubes, Journal of Materials Processing Technology, (v63), pp. 254-259. (2017). 